\title{
Positive bound state solutions for some Schrödinger-Poisson systems
}

\author{
Giovanna Cerami* Riccardo Molle ${ }^{\dagger}$
}

\begin{abstract}
The paper deals with a class of Schrödinger-Poisson systems, where the coupling term and the other coefficients do not have any symmetry property. Moreover, the setting we consider does not allow the existence of ground state solutions. Under suitable assumptions on the decay rate of the coefficients, we prove existence of a bound state, finite energy solution.
\end{abstract}

\section{Introduction}

In the last years there has been a large amount of work dealing with equations arising in Quantum Mechanics studied by means of variational tools. In this paper we focus on the following system

$$
\left\{\begin{array}{l}
-\Delta u+V(x) u+K(x) \phi(x) u=a(x)|u|^{p-1} u \\
-\Delta \phi=K(x) u^{2} \quad x \in \mathbb{R}^{3} .
\end{array}\right.
$$

According to a classical model, the interaction of a charge particle with an electromagnetic field can be described by coupling nonlinear Schrödinger and Maxwell equations. Systems like $(S P)$ are usually known as Schrödinger-Poisson systems and have been introduced in $[6,7]$ as a model to study electrostatic situations, that is cases in which the interaction between an electrostatic field and solitary waves for nonlinear stationary equations of Schrödinger type has to be considered. In $(S P)$ the first equation, which is a nonlinear stationary Schrödinger equation, is coupled with a Poisson equation to be satisfied by $\phi$, meaning that the potential feels the effect of the charge of the wave itself.

It is well known that, dealing with $(S P)$, one has to face different kinds of difficulties, which are related to the potential and to the unboundedness of the space $\mathbb{R}^{3}$. Many researches at the beginning have been devoted to the autonomous case and to the case

\footnotetext{
*Dipartimento di Matematica, Politecnico di Bari, Via Amendola 126/B - 70126 Bari, Italia. Email: cerami@poliba.it

†Dipartimento di Matematica, Università di Roma "Tor Vergata", Via della Ricerca Scientifica $n^{o}$ 1 - 00133 Roma. E-mail: molle@mat.uniroma2.it
} 
in which the coefficients are supposed radial (see f.i.[2] [3][20]), just to overcome the lack of compactness taking advantage of the compact embedding of the subspace of $H^{1}\left(\mathbb{R}^{3}\right)$ consisting of radial functions in $L^{q}\left(\mathbb{R}^{3}\right), q \in[2,6)$. More recently, many contributions to $(S P)$ have been given also looking at cases in which no symmetry assumptions are given on the coefficients appearing in $(S P)$ (see f.i. $[1,15,16]$ ). Describing all the various and interesting contributions in both directions, without forgetting something, is not an easy matter. So we prefer to refer readers, who are interested in a quite rich (even if not exhaustive) bibliography, to the recent paper [8].

However, it is worth observing that in most papers, the search of the positive, physically meaningful, solutions to $(S P)$, has been carried out looking for ground state solutions. Here we consider a non symmetric situation that has to be studied in a different way, because the assumptions imply nonexistence of ground state solutions to $(S P)$. Our aim is to show that even in this case a positive solution, of course at a higher energy level than the ground state level, exists.

We assume that:

$\left(H_{V}\right) V(x)=V_{\infty}+W(x)$ where $V_{\infty} \in \mathbb{R}^{+} \backslash\{0\}$ and $W(x) \in L^{3 / 2}\left(\mathbb{R}^{3}\right)$ is a nonnegative function such that

$$
\int_{\mathbb{R}^{3}} W(x)\left(|x| e^{2 \sqrt{V_{\infty}}|x|}\right) d x<\infty
$$

$\left(H_{K}\right) K \in L^{2}\left(\mathbb{R}^{3}\right)$ is a nonnegative function, $K \not \equiv 0$, such that for some $\eta>2 \sqrt{V_{\infty}}$ and $c, \bar{R}>0$

$$
K(x) \leq c e^{-\eta|x|} \quad x \in \mathbb{R}^{3},|x|>\bar{R}
$$

$\left(H_{a}\right) a(x)=a_{\infty}-\alpha(x)$, where $a_{\infty} \in \mathbb{R}^{+} \backslash\{0\}$ and $\alpha$ is function such that $0 \leq \alpha<a_{\infty}$ and

$$
\int_{\mathbb{R}^{3}} \alpha(x)\left(|x| e^{2 \sqrt{V_{\infty}}|x|}\right) d x<\infty .
$$

The result we obtain is stated in the following theorem:

Theorem 1.1 Assume that $\left(H_{V}\right),\left(H_{K}\right),\left(H_{a}\right)$, hold and that $p \in(3,5)$. Then $(S P)$ has a bound state solution $(u, \phi) \in H^{1}\left(\mathbb{R}^{3}\right) \times \mathcal{D}^{1,2}\left(\mathbb{R}^{3}\right)$ whose components are positive functions.

We remark that Theorem 1.1 generalizes to Schrödinger-Poisson systems well known, celebrated results proven in [5] for nonlinear Schrödinger equations. Actually in [5] problems like

$$
\left\{\begin{array}{l}
-\Delta u+V(x) u=a(x)|u|^{p-1} u \quad \text { in } \mathbb{R}^{N} \\
u(x) \rightarrow 0 \quad \text { as }|x| \rightarrow \infty .
\end{array}\right.
$$

are considered and, under similar conditions to $\left(H_{V}\right),\left(H_{a}\right)$, the existence of a positive not ground state solution is shown by using variational methods together deep algebraic 
topology tools. The proof of Theorem 1.1 takes advantage of some ideas introduced in [5] and in [4], but the argument, which does not need Homology theory, is more in the spirit of the one displayed in [13] and [12] to obtain existence and multiplicity results to $(S E)$, either in $\mathbb{R}^{N}$ either in exterior domains, when $a(x)$ is a constant. However here, because of the presence of the nonlocal term and the coefficient $a(x)$, the variational framework is different: we consider the functional related to $(S P)$ constrained on the corresponding Nehari manifold (instead of $\int_{\mathbb{R}^{3}}\left(|\nabla u|^{2}+V(x) u^{2}\right) d x$ constrained on the $L^{p}$ unitary sphere). Furthermore new delicate estimates, concerning the nonlocal term behaviour, are in order to show that the hopefully critical level of the energy functional belongs to an interval in which the compactness holds.

Lastly, we mention that, as far as we know, a similar topological situation related to $(S P)$ has been considered only in [15], where an existence theorem has been proved considering $V$ constant and assuming the $L^{2}$ norm of $K$ bounded by a suitably small number depending on the infimum of $a(x)$ and on the ground state level of the so called limit problem related to $(S P)$. Our theorem is obtained without imposing conditions of this kind, but just assuming on the coefficients suitable decay rate, that, on the other hand, are not requested in [15]. So in some sense our present result complements (and it is not comparable with) the above mentioned one.

The proof of Theorem 1.1 in contained in section 3, while section 2 is devoted to the costruction of the variational framework, to recall some known results and to state and prove some useful estimate.

\section{Notations, preliminary results and variational frame- work}

Hereafter we use the following notation:

- $H^{1}\left(\mathbb{R}^{3}\right)$ is the usual Sobolev space endowed with the standard scalar product and norm

$$
(u, v)=\int_{\mathbb{R}^{3}}[\nabla u \nabla v+u v] d x ; \quad\|u\|^{2}=\int_{\mathbb{R}^{3}}\left[|\nabla u|^{2}+u^{2}\right] d x .
$$

- $\mathcal{D}^{1,2}\left(\mathbb{R}^{3}\right)$ is the completion of $C_{0}^{\infty}\left(\mathbb{R}^{3}\right)$ with respect to the norm

$$
\|u\|_{\mathcal{D}^{1,2}}^{2}=\int_{\mathbb{R}^{3}}|\nabla u|^{2} d x .
$$

- $L^{q}(\Omega), 1 \leq q \leq+\infty, \Omega \subseteq \mathbb{R}^{3}$, denotes a Lebesgue space, the norm in $L^{q}(\Omega)$ is denoted by $|u|_{q, \Omega}$ when $\Omega$ is a proper subset of $\mathbb{R}^{3}$, by $|\cdot|_{p}$ when $\Omega=\mathbb{R}^{3}$.

- For any $\rho>0$ and for any $z \in \mathbb{R}^{3}, B(z, \rho)$ denotes the ball of radius $\rho$ centered at $z, \quad(\cdot \mid \cdot)_{\mathbb{R}^{3}}$ denotes the scalar product in $\mathbb{R}^{3}$, and for any measurable set $\mathcal{O} \subset \mathbb{R}^{3},|\mathcal{O}|$ denotes its Lebesgue measure. 
- $S$ is the best Sobolev constant that is

$$
S=\inf _{u \in H^{1}\left(\mathbb{R}^{3}\right) \backslash\{0\}} \frac{\|u\|^{2}}{|u|_{6}^{2}}=\inf _{u \in \mathcal{D}^{1,2}\left(\mathbb{R}^{3}\right) \backslash\{0\}} \frac{\|u\|_{\mathcal{D}^{1,2}}^{2}}{|u|_{6}^{2}} .
$$

- $c, c^{\prime}, C, C^{\prime}, C_{i}$ denote various positive constants.

In what follows, without any loss of generality we assume $V_{\infty}=a_{\infty}=1$.

It is known (see $[7,15])$ that solutions of $(\mathrm{SP})$ correspond to critical points of the functional $F: H^{1}\left(\mathbb{R}^{3}\right) \times \mathcal{D}^{1,2}\left(\mathbb{R}^{3}\right) \rightarrow \mathbb{R}$ defined by

$$
\begin{aligned}
F(u, \phi)= & \frac{1}{2} \int_{\mathbb{R}^{3}}\left(|\nabla u|^{2}+(V(x)+K(x) \phi) u^{2}\right) d x-\frac{1}{4} \int_{\mathbb{R}^{3}}|\nabla \phi|^{2} d x \\
& -\frac{1}{p+1} \int_{\mathbb{R}^{3}} a(x)|u|^{p+1} d x .
\end{aligned}
$$

It is also not difficult to show that (SP) can be reduced to a single equation with a nonlocal term. Indeed, considering for all $u \in H^{1}\left(\mathbb{R}^{3}\right)$, the linear functional $L_{u}$ defined in $\mathcal{D}^{1,2}\left(\mathbb{R}^{3}\right)$ by

$$
L_{u}(v)=\int_{\mathbb{R}^{3}} K(x) u^{2} v d x
$$

Hölder and Sobolev inequalities imply

$$
\left|L_{u}(v)\right| \leq S^{-1}|K|_{2}|u|_{6}^{2} \cdot\|v\|_{\mathcal{D}^{1,2}}
$$

and, then, Lax-Milgram theorem gives, for all $u \in H^{1}\left(\mathbb{R}^{3}\right)$, the existence of a unique positive function, $\phi_{u} \in \mathcal{D}^{1,2}\left(\mathbb{R}^{3}\right)$, which is weak solution of

$$
-\Delta \phi=K(x) u^{2} \quad x \in \mathbb{R}^{3} .
$$

Therefore, substituting $\phi_{u}$ in the first equation of (SP) we are led to the equation

$$
-\Delta u+V(x) u+K(x) \phi_{u} u=a(x)|u|^{p-1} u,
$$

and solutions of (SP) can be searched as critical points of the "reduced" functional

$$
I(u)=\frac{1}{2} \int_{\mathbb{R}^{3}}\left(|\nabla u|^{2}+V(x) u^{2}\right) d x+\frac{1}{4} \int_{\mathbb{R}^{3}} K(x) \phi_{u}(x) u^{2} d x-\frac{1}{p+1} \int_{\mathbb{R}^{3}} a(x)|u|^{p+1} d x .
$$

Let $\Phi: H^{1}\left(\mathbb{R}^{3}\right) \rightarrow \mathcal{D}^{1,2}\left(\mathbb{R}^{3}\right)$ be the operator defined by

$$
\Phi(u)=\phi_{u},
$$

next two propositions collect some properties of it. 


\section{Proposition $2.1 \quad$ 1) $\Phi$ is continuous;}

2) $\Phi$ maps bounded sets into bounded sets;

3) $\Phi(t u)=t^{2} \Phi(u)$ for all $t \in \mathbb{R}$;

4) the following representation formula holds

$$
\Phi(u)=\frac{1}{4 \pi} \int_{\mathbb{R}^{3}} \frac{K(y)}{|x-y|} u^{2}(y) d y=\frac{1}{4 \pi} \frac{1}{|x|} * K(x) u^{2} .
$$

The proof of (1) and (2) can be found in [15]. Properties (3) and (4) are straight consequence of the definition of $\Phi(u)$ as solution of (2.2).

Proposition 2.2 Let assume $u_{n} \rightarrow u$ in $H^{1}\left(\mathbb{R}^{3}\right)$. Then

a) $\Phi\left(u_{n}\right) \rightarrow \Phi(u)$ in $\mathcal{D}^{1,2}\left(\mathbb{R}^{3}\right)$;

b) $\int_{\mathbb{R}^{3}} K(x) \phi_{u_{n}} u_{n}^{2} d x \rightarrow \int_{\mathbb{R}^{3}} K(x) \phi_{u} u^{2} d x ;$

c) $\int_{\mathbb{R}^{3}} K(x) \phi_{u_{n}} u_{n} \varphi d x \rightarrow \int_{\mathbb{R}^{3}} K(x) \phi_{u} u \varphi d x \quad \forall \varphi \in H^{1}\left(\mathbb{R}^{3}\right)$.

Proof (a) Since, by definition of $\Phi$, for all $u \in H^{1}\left(\mathbb{R}^{3}\right)$

$$
\|\Phi(u)\|_{\mathcal{D}^{1,2}}=\left\|L_{u}\right\|_{\mathcal{L}\left(\mathcal{D}^{1,2}, \mathbb{R}\right)},
$$

to prove (a) we intend to show that, as $n \rightarrow \infty$

$$
\left\|L_{u_{n}}-L_{u}\right\|_{\mathcal{L}\left(\mathcal{D}^{1,2}, \mathbb{R}\right)} \rightarrow 0 .
$$

Let $\varepsilon>0$ be fixed arbitrarily, then there exists a positive number $R_{\varepsilon}$ so large that $|K|_{L^{2}\left(\mathbb{R}^{3} \backslash B\left(0, R_{\varepsilon}\right)\right)}<\varepsilon$. Thus, for all $v \in \mathcal{D}^{1,2}\left(\mathbb{R}^{3}\right)$ we have

$$
\begin{aligned}
\left|L_{u_{n}}(v)-L_{u}(v)\right| & =\int_{\mathbb{R}^{3}} K\left(u_{n}^{2}-u^{2}\right) v d x \\
& \leq \int_{\mathbb{R}^{3} \backslash B\left(0, R_{\varepsilon}\right)} K\left|u_{n}^{2}-u^{2}\right||v| d x+\int_{B\left(0, R_{\varepsilon}\right)} K\left|u_{n}^{2}-u^{2}\right||v| d x \\
& \leq|K|_{L^{2}\left(\mathbb{R}^{3} \backslash B\left(0, R_{\varepsilon}\right)\right)}\left|u_{n}^{2}-u^{2}\right|_{3}|v|_{6}+\left(\int_{B\left(0, R_{\varepsilon}\right)} K^{\frac{6}{5}}\left|u_{n}^{2}-u^{2}\right|^{\frac{6}{5}} d x\right)^{\frac{5}{6}}|v|_{6} \\
& \leq\left(\varepsilon c+\left(\int_{B\left(0, R_{\varepsilon}\right)} K^{\frac{6}{5}}\left|u_{n}+u\right|^{\frac{6}{5}}\left|u_{n}-u\right|^{\frac{6}{5}} d x\right)^{\frac{5}{6}}\right)\|v\|_{\mathcal{D}^{1,2} .}
\end{aligned}
$$

Therefore, to obtain (2.6) we just need to see that the last integral in (2.7) goes to 0 as $n \rightarrow \infty$. Let us set $B_{M}=\left\{x \in B\left(0, R_{\varepsilon}\right): K(x)>M\right\}$ and remark that being 
$K \in L^{2}\left(\mathbb{R}^{3}\right),\left|B_{M}\right| \rightarrow 0$ as $M \rightarrow \infty$. So, for large $M,\left(\int_{B_{M}} K^{2}\right)^{\frac{3}{5}}<\varepsilon$. Furthermore, we can assume that, up to a subsequence, $u_{n} \rightarrow u$ in $L_{\text {loc }}^{\frac{12}{5}}\left(\mathbb{R}^{3}\right)$. Hence, we obtain

$$
\begin{aligned}
& \int_{B\left(0, R_{\varepsilon}\right)} K^{\frac{6}{5}}\left|u_{n}+u\right|^{\frac{6}{5}}\left|u_{n}-u\right|^{\frac{6}{5}} d x \\
= & \int_{B_{M}} K^{\frac{6}{5}}\left|u_{n}+u\right|^{\frac{6}{5}}\left|u_{n}-u\right|^{\frac{6}{5}} d x+\int_{B\left(0, R_{\varepsilon}\right) \backslash B_{M}} K^{\frac{6}{5}}\left|u_{n}+u\right|^{\frac{6}{5}}\left|u_{n}-u\right|^{\frac{6}{5}} d x \\
\leq & \left(\int_{B_{M}} K^{2} d x\right)^{\frac{3}{5}}\left(\int_{\mathbb{R}^{3}}\left|u_{n}+u\right|^{6} d x\right)^{\frac{1}{5}}\left(\int_{\mathbb{R}^{3}}\left|u_{n}-u\right|^{\frac{1}{5}} d x\right)^{\frac{1}{2}}\left(\int_{B\left(0, R_{\varepsilon}\right)}\left|u_{n}-u\right|^{\frac{12}{5}} d x\right)^{\frac{1}{2}} \\
& +M^{\frac{6}{5}}\left(\int_{B\left(0, R_{\varepsilon}\right)}\left|u_{n}+u\right|^{\frac{12}{5}} d x\right)^{\frac{1}{2}}(c \varepsilon+c o(1),
\end{aligned}
$$

as desired.

(b) To show (b) we write

$$
\int_{\mathbb{R}^{3}}\left(K \phi_{u_{n}} u_{n}^{2}-K \phi_{u} u^{2}\right) d x=\int_{\mathbb{R}^{3}} K\left(u_{n}^{2}-u^{2}\right) \phi_{u_{n}} d x+\int_{\mathbb{R}^{3}} K u^{2}\left(\phi_{u_{n}}-\phi_{u}\right) d x .
$$

and we observe that

$$
\int_{\mathbb{R}^{3}} K u^{2}\left(\phi_{u_{n}}-\phi_{u}\right) d x \leq|K|_{2}|u|_{6}^{2}\left|\phi_{u_{n}}-\phi_{u}\right|_{6}=o(1)
$$

because, by $(a)$, as $n \rightarrow \infty \phi_{u_{n}} \rightarrow \phi_{u}$ in $\mathcal{D}^{1,2}\left(\mathbb{R}^{3}\right)$ and, then, in $L^{6}\left(\mathbb{R}^{3}\right)$. To verify that, as $n \rightarrow \infty, \quad \int_{\mathbb{R}^{3}} K\left(u_{n}^{2}-u^{2}\right) \phi_{u_{n}} d x \longrightarrow 0$ holds true, one can repeat the argument used in the proof of $(a)$, obviously replacing $v$ by $\phi_{u_{n}}$ and taking into account that the sequence $\left(\left|\phi_{u_{n}}\right|_{6}\right)_{n}$ is bounded.

(c) Writing

$$
\int_{\mathbb{R}^{3}}\left(K \phi_{u_{n}} u_{n} \varphi-K \phi_{u} u \varphi\right) d x=\int_{\mathbb{R}^{3}}\left(K \varphi u_{n}\right)\left(\phi_{u_{n}}-\phi_{u}\right) d x+\int_{\mathbb{R}^{3}}\left(K \varphi \phi_{u}\right)\left(u_{n}-u\right) d x,
$$

the claim straightly follows from $(a)$ and the assumption on $\left(u_{n}\right)_{n}$.

Remark 2.3 One of the referees has drawn to our attention that part b) of previous Lemma seems could be also obtained as a consequence of a nonlocal version, recently proved in a still now unpublished paper [19], of the well known Brezis-Lieb lemma.

Furthermore, we mention two other papers [10] and [11], treating a problem formally similar to $(S P)$, whose interest has been pointed out to us by the same referee. 
It is not difficult to verify that the functional $I$ is bounded neither from below, nor from above. So it is convenient to consider $I$ restricted to a natural constraint: the Nehari manifold.

We set

$$
\mathcal{N}=\left\{u \in H^{1}\left(\mathbb{R}^{3}\right) \backslash\{0\}: I^{\prime}(u)[u]=0\right\}
$$

and we remark that, for all $u \in \mathcal{N}$, we can write

$$
\begin{aligned}
I_{\left.\right|_{\mathcal{N}}}(u) & =\left(\frac{1}{2}-\frac{1}{p+1}\right) \int_{\mathbb{R}^{3}}\left(|\nabla u|^{2}+V(x) u^{2}\right) d x+\left(\frac{1}{4}-\frac{1}{p+1}\right) \int_{\mathbb{R}^{3}} K(x) \phi_{u}(x) u^{2} d x \\
& =\frac{1}{4} \int_{\mathbb{R}^{3}}\left(|\nabla u|^{2}+V(x) u^{2}\right) d x+\left(\frac{1}{4}-\frac{1}{p+1}\right) \int_{\mathbb{R}^{3}} a(x)|u|^{p+1} d x,
\end{aligned}
$$

from which one easily deduces that $I$ is bounded from below on $\mathcal{N}$. Furthermore, for all $u \in H^{1}\left(\mathbb{R}^{3}\right) \backslash\{0\}$, there exists a unique $t_{u} \in \mathbb{R}^{+} \backslash\{0\}$ such that $t_{u} u \in \mathcal{N}$. Indeed, $t_{u}$ must satisfy the equation

$$
\begin{aligned}
0=I^{\prime}(t u)[t u]= & t^{2}\left(\|u\|^{2}+\int_{\mathbb{R}^{3}} W(x) u^{2} d x\right)+t^{4} \int_{\mathbb{R}^{3}} K(x) \phi_{u}(x) u^{2} d x \\
& -t^{p+1} \int_{\mathbb{R}^{3}} a(x)|u|^{p+1} d x
\end{aligned}
$$

which has a unique positive solution because $p>3$. The function $t_{u} u \in \mathcal{N}$ is called the projection of $u$ on $\mathcal{N}$ and we also point out that

$$
I\left(t_{u} u\right)=\max _{t>0} I(t u)
$$

Actually, more precise information is available on $\mathcal{N}$ and $I_{\mid \mathcal{N}}$ and it can be summarized in the following lemma, whose proof can be found in [15]:

Lemma $2.4 \quad$ 1) $\mathcal{N}$ is a $C^{1}$ regular manifold diffeomorphic to the sphere of $H^{1}\left(\mathbb{R}^{3}\right)$;

2) I is bounded from below on $\mathcal{N}$ by a positive constant;

3) $u$ is a free critical point of $I$ if and only if $u$ is a critical point of I constrained on $\mathcal{N}$.

For what follows it is also useful to introduce the problem

$$
\left\{\begin{array}{l}
-\Delta u+u=|u|^{p-1} u \quad x \in \mathbb{R}^{3} \quad\left(P_{\infty}\right) \\
u \in H^{1}\left(\mathbb{R}^{3}\right)
\end{array}\right.
$$

that can be called the "problem at infinity" related to $(\mathrm{SP}) .\left(P_{\infty}\right)$ has been widely studied and combining results of [9], [17],[18] the following Proposition can be proved: 
Proposition $2.5\left(P_{\infty}\right)$ has a positive, ground state, solution $w \in H^{1}\left(\mathbb{R}^{3}\right)$, which is radially symmetric about the origin, unique up to translations, and such that

$$
\lim _{|x| \rightarrow+\infty}\left|D^{s} w(x)\right|\left(|x| e^{|x|}\right)=d_{s}>0 \quad s=0,1 .
$$

Throughout the paper we denote by $I_{\infty}: H^{1}\left(\mathbb{R}^{3}\right) \rightarrow \mathbb{R}$ the functional whose critical points are solutions of $\left(P_{\infty}\right)$, that is

$$
I_{\infty}(u)=\frac{1}{2}\|u\|^{2}-\frac{1}{p+1} \int_{\mathbb{R}^{3}}|u|^{p+1} d x
$$

and by $\mathcal{N}_{\infty}$ the corresponding Nehari manifold

$$
\mathcal{N}_{\infty}=\left\{u \in H^{1}\left(\mathbb{R}^{3}\right) \backslash\{0\}:\|u\|^{2}=|u|_{p+1}^{p+1}\right\} .
$$

As for $\mathcal{N}, \quad \mathcal{N}_{\infty}$ turns out to be a $C^{1}$ manifold diffeomorphic to the unitary sphere of $H^{1}\left(\mathbb{R}^{3}\right)$, so to any function $u \in H^{1}\left(\mathbb{R}^{3}\right) \backslash\{0\}$ there corresponds a unique function $\tau_{u} u \in \mathcal{N}_{\infty}$ such that

$$
I_{\infty}\left(\tau_{u} u\right)=\max _{t>0} I_{\infty}(t u) .
$$

Such a function is called the projection of $u$ on $\mathcal{N}_{\infty}$. We remark that

$$
I_{\infty}(u)=\left(\frac{1}{2}-\frac{1}{p+1}\right)\|u\|^{2}=\left(\frac{1}{2}-\frac{1}{p+1}\right)|u|_{p+1}^{p+1} \quad \forall u \in \mathcal{N}_{\infty} ;
$$

moreover, in what follows we use the notation

$$
m_{\infty}:=\min _{\mathcal{N}_{\infty}} I_{\infty}=I_{\infty}(w)=\left(\frac{1}{2}-\frac{1}{p+1}\right)\|w\|^{2}=\left(\frac{1}{2}-\frac{1}{p+1}\right)|w|_{p+1}^{p+1} .
$$

Remark 2.6 It is worth observing that any changing sign solution $\bar{u}$ of $\left(P_{\infty}\right)$ is such that $I_{\infty}(\bar{u})>2 m_{\infty}$, see f.i. [22].

Actually, in our setting we need just the weak inequality $I_{\infty}(\bar{u}) \geq 2 m_{\infty}$, that is quite easy to show. Indeed, assume $\bar{u}=\bar{u}^{+}-\bar{u}^{-}$and $I_{\infty}^{\prime}(\bar{u})[\bar{u}]=\|\bar{u}\|^{2}-|\bar{u}|_{p+1}^{p+1}=0$. Then $0=\left\|\bar{u}^{+}\right\|^{2}-\left|\bar{u}^{+}\right|_{p+1}^{p+1}=I_{\infty}^{\prime}(\bar{u})\left[\bar{u}^{+}\right]=I_{\infty}^{\prime}\left(\bar{u}^{+}\right)\left[\bar{u}^{+}\right]$, that is $\bar{u}^{+} \in \mathcal{N}_{\infty}$, and, then $I_{\infty}\left(\bar{u}^{+}\right) \geq m_{\infty}$. Analogously one sees that $I_{\infty}\left(\bar{u}^{-}\right) \geq m_{\infty}$, thus $I_{\infty}(\bar{u}) \geq 2 m_{\infty}$.

Problem $(S P)$ exhibit a lack of compactness, whose origin is the invariance of $\mathbb{R}^{3}$ under the action of the noncompact group of translations, and which appears in the fact that sequences of functions of $\mathcal{N}$ along which $I$ satisfies the Palais-smale condition can be not relatively compact. The following "splitting" lemma for Palais-Smale sequences point out the importance of the problem at infinity in locating these "bad" levels: 
Lemma 2.7 Let $\left(u_{n}\right)_{n}$ be a $(P S)$ sequence of I constrained on $\mathcal{N}$, i.e. $u_{n} \in \mathcal{N}$ and

a) $I\left(u_{n}\right)$ is bounded;

b) $\nabla I_{\left.\right|_{\mathcal{N}}}\left(u_{n}\right) \rightarrow 0$ strongly in $H^{1}\left(\mathbb{R}^{3}\right)$.

Then there exist a solution $\bar{u}$ of (2.3), a number $k \in \mathbb{N} \cup\{0\}, k$ functions $u^{1}, \ldots, u^{k}$ of $H^{1}\left(\mathbb{R}^{3}\right)$ and $k$ sequences of points $\left(y_{n}^{j}\right), y_{n}^{j} \in \mathbb{R}^{3}, 1 \leq j \leq k$ such that, up to a subsequence,

(i) $\left|y_{n}^{j}\right| \rightarrow+\infty,\left|y_{n}^{j}-y_{n}^{i}\right| \rightarrow+\infty$ if $i \neq j, \quad n \rightarrow+\infty$;

(ii) $u_{n}-\sum_{j=1}^{k} u^{j}\left(\cdot-y_{n}^{j}\right) \longrightarrow \bar{u}$, in $H^{1}\left(\mathbb{R}^{3}\right)$;

(iii) $I\left(u_{n}\right) \rightarrow I(\bar{u})+\sum_{j=1}^{k} I_{\infty}\left(u^{j}\right)$;

(iv) $u^{j}$ are non trivial weak solutions of $\left(P_{\infty}\right)$.

Moreover, we agree that in the case $k=0$ the above holds without $u^{j}$.

The above lemma can be proved arguing as in [15] and taking advantage of Proposition 2.2 .

We end this section with two technical lemmas that are basic for some estimates in next section. For the proof of Lemma 2.8 we refer to [13], while, for reader's convenience, we give a sketchy proof of Lemma 2.9, which is in the spirit of Proposition 1.2 of $[4]$.

Lemma 2.8 For all $a, b \in \mathbb{R}^{+}$, for all $p \geq 1$, the following relation holds true

$$
(a+b)^{p+1} \geq a^{p+1}+b^{p+1}+p\left(a^{p} b+a b^{p}\right) .
$$

Lemma 2.9 If $g \in L^{\infty}\left(\mathbb{R}^{3}\right)$ and $h \in L^{1}\left(\mathbb{R}^{3}\right)$ are such that, for some $\alpha \geq 0, b \geq 0$, $\gamma \in \mathbb{R}$

$$
\lim _{|x| \rightarrow+\infty} g(x) e^{\alpha|x|}|x|^{b}=\gamma
$$

and

$$
\int_{\mathbb{R}^{3}}|h(x)| e^{\alpha|x|}|x|^{b} d x<+\infty
$$

then, for every $z \in \mathbb{R}^{3} \backslash\{0\}$,

$$
\lim _{\rho \rightarrow+\infty}\left(\int_{\mathbb{R}^{3}} g(x+\rho z) h(x) d x\right) e^{\alpha|\rho z|}|\rho z|^{b}=\gamma \int_{\mathbb{R}^{3}} h(x) e^{-\alpha\left[(x \mid z)_{\mathbb{R}^{3}} /|z|\right]} d x .
$$


Proof Considering (2.18) and the relation

$$
\lim _{\rho \rightarrow+\infty} \frac{e^{\alpha|\rho z|}}{e^{\alpha|x+\rho z|}}=e^{-\alpha\left[(x \mid z)_{\mathbb{R}^{3}} /|z|\right]}
$$

we deduce

$$
\lim _{\rho \rightarrow+\infty} g(x+\rho z) h(x) e^{\alpha|\rho z|}|\rho z|^{b}=\gamma h(x) e^{-\alpha\left[(x \mid z)_{\mathbb{R}^{3}} /|z|\right]}
$$

pointwise for all $x \in \mathbb{R}^{3}$.

On the other hand, by using (2.18) and assumption $g \in L^{\infty}\left(\mathbb{R}^{3}\right)$, we obtain the uniform estimate

$$
\begin{aligned}
\left.\left|g(x+\rho z) h(x) e^{\alpha|\rho z|}\right| \rho z\right|^{b} \mid & \leq c\left(e^{-\alpha|x+\rho z|}(1+|x+\rho z|)^{-b}\right)|h(x)| e^{\alpha|\rho z|}|\rho z|^{b} \\
& \leq c|h(x)| e^{\alpha|x|}(1+|x+\rho z|)^{-b}|\rho z|^{b} \\
& \leq c|h(x)| e^{\alpha|x|}\left(1+|x|^{b}\right)
\end{aligned}
$$

where the r.h.s. is integrable by (2.19). Then the statement follows by the dominated convergence theorem.

q.e.d.

\section{Existence of a solution to $(S P)$}

This section is devoted to the proof of our main result. We first show that $(S P)$ cannot be solved by minimization. Then, we prove the existence of a higher energy solution, by showing that there is a change of topology between two sublevel sets of the functional $I$ and that this change, thanks to compactness arguments, implies the existence of a critical point of I.

Throughout this section we suppose that assumptions of Theorem 1.1 are satisfied.

Proposition 3.1 The relation $\inf _{\mathcal{N}} I=m_{\infty}$ holds and the infimum is not achieved.

Proof Set $m:=\inf _{\mathcal{N}} I$ and remark that, by Lemma $2.4, m>0$.

Let $u \in \mathcal{N}$ be arbitrarily chosen, and let $\tau_{u} u \in \mathcal{N}_{\infty}$ be its projection on $\mathcal{N}_{\infty}$. Taking into account that $\phi_{u}, W, K, \alpha$ are nonnegative functions and using (2.11), we get

$$
I(u) \geq I\left(\tau_{u} u\right) \geq \frac{1}{2}\left\|\tau_{u} u\right\|^{2}-\frac{1}{p+1} \int_{\mathbb{R}^{3}}\left|\tau_{u} u\right|^{p+1} d x=I_{\infty}\left(\tau_{u} u\right) \geq m_{\infty},
$$

from which

$$
m_{\infty} \leq m
$$


follows. To prove the reverse inequality let us consider the sequence $\left(u_{n}\right)_{n}, u_{n}=t_{n} w_{n} \in$ $\mathcal{N}$, where $w_{n}(\cdot)=w\left(\cdot-z_{n}\right)$, being $w$ the ground state positive solution of $\left(P_{\infty}\right),\left(z_{n}\right)_{n}$ a sequence of points in $\mathbb{R}^{3}$ such that $\left|z_{n}\right| \rightarrow+\infty$, as $n \rightarrow+\infty$, and $t_{n}=t_{w_{n}}$. Let us show that

$$
\lim _{n \rightarrow+\infty} I\left(u_{n}\right)=m_{\infty}
$$

Indeed, since $w_{n}$ weakly converges to zero in $H^{1}\left(\mathbb{R}^{3}\right)$, (b) of Proposition 2.2 implies

$$
\lim _{n \rightarrow+\infty} \int_{\mathbb{R}^{3}} K(x) \phi_{w_{n}}(x) w_{n}^{2} d x=0
$$

moreover, using $\left(H_{V}\right)$ one readily verifies that

$$
\lim _{n \rightarrow+\infty} \int_{\mathbb{R}^{3}} W(x) w_{n}^{2} d x=0 .
$$

Therefore, thanks to (2.9), in order to obtain (3.2) we just need to show that $t_{n} \rightarrow 1$, as $n \rightarrow+\infty$. To this end, using (2.10), we write

$$
\|w\|^{2}=\left\|w_{n}\right\|^{2}=-\int_{\mathbb{R}^{3}} W(x) w_{n}^{2} d x-t_{n}^{2} \int_{\mathbb{R}^{3}} K(x) \phi_{w_{n}}(x) w_{n}^{2} d x+t_{n}^{p-1} \int_{\mathbb{R}^{3}} a(x)\left|w_{n}\right|^{p+1} d x,
$$

from which observing that, by $\left(H_{a}\right)$,

$$
\lim _{n \rightarrow+\infty} \int_{\mathbb{R}^{3}} a(x)\left|w_{n}\right|^{p+1} d x=\int_{\mathbb{R}^{3}}\left|w_{n}\right|^{p+1} d x,
$$

that $\|w\|^{2}=|w|_{p+1}^{p+1}$, and again using (3.3), (3.4), we deduce that $\left(t_{n}\right)_{n}$ is bounded, bounded away from zero and, actually, $\lim _{n \rightarrow \infty} t_{n}=1$.

To complete the proof, we argue by contradiction and we assume that $\bar{u} \in \mathcal{N}$ exists such that $I(\bar{u})=m=m_{\infty}$. Denoting by $\tau_{\bar{u}} \bar{u}$ its projection on $\mathcal{N}_{\infty}$, we have

$$
\begin{aligned}
m_{\infty} & \leq I_{\infty}\left(\tau_{\bar{u}} \bar{u}\right)=\left(\frac{1}{2}-\frac{1}{p+1}\right)\left\|\tau_{\bar{u}} \bar{u}\right\|^{2} \\
& \leq\left(\frac{1}{2}-\frac{1}{p+1}\right)\left\|\tau_{\bar{u}} \bar{u}\right\|^{2}+\left(\frac{1}{4}-\frac{1}{p+1}\right) \int_{\mathbb{R}^{3}} K(x) \phi_{\tau_{\bar{u}} \bar{u}}(x)\left(\tau_{\bar{u}} \bar{u}\right)^{2} d x \\
& =I\left(\tau_{\bar{u}} \bar{u}\right) \leq I(\bar{u})=m=m_{\infty},
\end{aligned}
$$

so we infer $\tau_{\bar{u}}=1$, and

$$
\int_{\mathbb{R}^{3}} K(x) \phi_{\bar{u}}(x) \bar{u}^{2} d x=0 .
$$

Thus, $\bar{u} \in \mathcal{N}_{\infty}$ and $I_{\infty}(\bar{u})=m_{\infty}$. Therefore, by the uniqueness of the family realizing $m_{\infty}$, some $z \in \mathbb{R}^{3}$ must exist for which

$$
\bar{u}(x)=w(x-z)>0, \quad \forall x \in \mathbb{R}^{3},
$$


holds true. The above equality implies $\int_{\mathbb{R}^{3}} K(x) \phi_{\bar{u}}(x) \bar{u}^{2} d x>0$, that contradicts (3.5).

Proposition 3.1 combined with Lemma 2.7 and Remark 2.6 allows us to locate an interval of values of $I$ in which the compactness holds.

Corollary 3.2 The functional I constrained on $\mathcal{N}$ satisfies the Palais-Smale condition in $\left(m_{\infty}, 2 m_{\infty}\right)$.

Moreover, if $\left(u_{n}\right)_{n}$ is a $(P S)_{m_{\infty}}$ sequence, then, up to a subsequence,

$$
u_{n}(x)=w\left(x-z_{n}\right)+o(1),
$$

being $z_{n} \in \mathbb{R}^{3},\left|z_{n}\right| \rightarrow+\infty$ and $w$ the positive ground state solution of $\left(P_{\infty}\right)$.

Proof Let $\left(u_{n}\right)_{n}$ be a P-S sequence of $I_{\mid \mathcal{N}}$ such that $I\left(u_{n}\right) \rightarrow c \in\left[m_{\infty}, 2 m_{\infty}\right)$ and apply Lemma 2.7. Then, the claim follows straightly recalling that any solution $u$ of $\left(P_{\infty}\right)$ verifies $I_{\infty}(u) \geq m_{\infty}$ and, if it changes sign, $I_{\infty}(u) \geq 2 m_{\infty}$ and, furthermore, that any critical point $v$ of $I$ is such that $I(v)>m_{\infty}$.

q.e.d.

Let us now remind the definition of barycenter of a function $u \in H^{1}\left(\mathbb{R}^{3}\right) \backslash\{0\}$, which has been introduced in [14]. We set

$$
\mu(u)(x)=\frac{1}{|B(0,1)|} \int_{B(x, 1)}|u(y)| d y,
$$

and we remark that $\mu(u)$ is bounded and continuous, so the function

$$
\hat{u}(x)=\left[\mu(u)(x)-\frac{1}{2} \max \mu(u)\right]^{+}
$$

is well defined, continuous, and has compact support. Thus, we can define $\beta: H^{1}\left(\mathbb{R}^{3}\right) \backslash$ $\{0\} \rightarrow \mathbb{R}^{3}$ as

$$
\beta(u)=\frac{1}{|\hat{u}|_{1}} \int_{\mathbb{R}^{3}} \hat{u}(x) x d x .
$$

$\beta$ is well defined, because $\hat{u}$ has compact support, and it is not difficult to verify that it enjoys the following properties:

$\beta$ is continuous in $H^{1}\left(\mathbb{R}^{3}\right) \backslash\{0\}$;

if $u$ is a radial function, then $\beta(u)=0$;

for all $t \neq 0$ and for all $u \in H^{1}\left(\mathbb{R}^{3}\right) \backslash\{0\}, \beta(t u)=\beta(u)$;

given $z \in \mathbb{R}^{3}$ and setting $u_{z}(x)=u(x-z), \beta\left(u_{z}\right)=\beta(u)+z$. 
Lemma 3.3 Set

$$
\mathcal{B}_{0}:=\inf \{I(u) \quad: \quad u \in \mathcal{N}, \quad \beta(u)=0\}
$$

Then,

$$
\mathcal{B}_{0}>m_{\infty}
$$

Proof Clearly $\mathcal{B}_{0} \geq m_{\infty}$. Suppose, by contradiction, $\mathcal{B}_{0}=m_{\infty}$. Then, a sequence $\left(\tilde{u}_{n}\right)_{n}$ such that $\tilde{u}_{n} \in \mathcal{N}, \beta\left(\tilde{u}_{n}\right)=0$ and $I\left(\tilde{u}_{n}\right) \rightarrow m_{\infty}$ exists. By the Ekeland variational principle, we can assert the existence of another sequence $\left(u_{n}\right)_{n}, u_{n} \in \mathcal{N}$, such that $I\left(u_{n}\right) \rightarrow m_{\infty}, \nabla I_{\left.\right|_{\mathcal{N}}}\left(u_{n}\right) \rightarrow 0$ and $\left\|u_{n}-\tilde{u}_{n}\right\| \rightarrow 0$. Hence, in view of $(3.8), \beta\left(u_{n}\right)=o(1)$.

On the other hand, by Corollary $3.2, u_{n}(x)=w\left(x-z_{n}\right)+o(1)$, where $\left(z_{n}\right)_{n} \in \mathbb{R}^{3}$ and $\left|z_{n}\right| \rightarrow \infty$. So we get

$$
o(1)=\beta\left(u_{n}\right)=\beta\left(w\left(x-z_{n}\right)\right)+o(1)=z_{n}+o(1)
$$

reaching a contradiction.

q.e.d.

Set now

$$
\Sigma=\left\{z \in \mathbb{R}^{3}:\left|z-e_{1}\right|=2\right\}
$$

where $e_{1}=(1,0,0)$.

In order to associate to any couple $(z, s) \in \Sigma \times[0,1]$ a function belonging to $\mathcal{N}$ and a function belonging to $\mathcal{N}_{\infty}$, we consider

$$
\bar{\psi}_{\rho}[z, s](x):=(1-s) w\left(x-\rho e_{1}\right)+s w(x-\rho z) \quad x \in \mathbb{R}^{3} .
$$

Then we denote by

$$
\begin{gathered}
\psi_{\rho}[z, s] \quad \text { the projection of } \bar{\psi}_{\rho}[z, s] \text { on } \mathcal{N}, \\
\psi_{\infty, \rho}[z, s] \quad \text { the projection of } \bar{\psi}_{\rho}[z, s] \text { on } \mathcal{N}_{\infty} .
\end{gathered}
$$

We remark that the definition of $\psi_{\rho}[z, s]$ and $\psi_{\infty, \rho}[z, s]$ implies, for any choice of $\rho>0,(z, s) \in \Sigma \times[0,1]$, the existence of positive numbers, $t_{\rho, z, s}:=t_{\bar{\psi}_{\rho}[z, s]}$ and $\tau_{\rho, z, s}:=\tau_{\bar{\psi}_{\rho}[z, s]}$ such that

$$
\psi_{\rho}[z, s]=t_{\rho, z, s} \bar{\psi}_{\rho}[z, s] ; \quad \psi_{\infty, \rho}[z, s]=\tau_{\rho, z, s} \bar{\psi}_{\rho}[z, s]
$$

Proposition $3.4 \quad$ a) $\beta\left(\psi_{\rho}[z, 1]\right)=\rho z$, for all $\rho>0$ and for all $z \in \Sigma$;

b) for every $\rho>0$, there exists $(\bar{z}, \bar{s}) \in \Sigma \times[0,1)$ such that $\beta\left(\psi_{\rho}[\bar{z}, \bar{s}]\right)=0$. 


\section{Proof}

a) Observe that $\bar{\psi}_{\rho}[z, 1](x)=w(x-\rho z)$, then by $(3.9)-(3.11)$ and $(3.13)$

$$
\beta\left(\psi_{\rho}[z, 1]\right)=\beta\left(\bar{\psi}_{\rho}[z, 1]\right)=\beta(w(\cdot-\rho z))=\beta(w(\cdot))+\rho z=\rho z .
$$

b) follows from a), the continuity of the maps $\beta$ and $\psi_{\rho}$, and the invariance of topological degree by homotopy. Indeed, considering for all $\rho>0$ the map $\mathcal{G}_{\rho}: \Sigma \times$ $[0,1] \rightarrow \mathbb{R}^{3}$ defined by $\mathcal{G}_{\rho}(z, s)=(1-s) \rho e_{1}+s \rho z$, taking into account a), we deduce $0 \neq d\left(\mathcal{G}_{\rho}, \Sigma \times[0,1), 0\right)=d\left(\beta \circ \psi_{\rho}, \Sigma \times[0,1), 0\right)$, thus the equation $\beta \circ \psi_{\rho}(z, s)=0$ must have a solution $(\bar{z}, \bar{s}) \in \Sigma \times[0,1)$.

q.e.d.

Proposition 3.5 There exists $\rho_{b} \in \mathbb{R}^{+} \backslash\{0\}$ such that

$$
\max \left\{I\left(\psi_{\rho}[z, 1]\right): z \in \Sigma\right\}<\mathcal{B}_{0} \quad \forall \rho>\rho_{b} .
$$

Proof Since $\psi_{\rho}[z, 1](x)=t_{\rho, z, 1} \bar{\psi}_{\rho}[z, 1](x)$, and $\bar{\psi}_{\rho}[z, 1]=w(\cdot-\rho z)$, one can obtain (3.14) by a quite analogous argument to that displayed to prove (3.2) in Proposition 3.1.

q.e.d.

Lemma 3.6 There exist $c, q_{\eta} \in \mathbb{R}^{+} \backslash\{0\}$ such that

$$
\int_{\mathbb{R}^{3}} K(x) \phi_{w(\cdot-\rho \zeta)}(x) w^{2}(x-\rho \zeta) d x \leq c e^{-\left(2+q_{\eta}\right) \rho},
$$

for all $\zeta \in \mathbb{R}^{3}:|\zeta| \geq 1$.

Proof Without any loss of generality we can suppose $|\zeta|=1$ and fix $\zeta=e_{1}$.

Let us set $q=\frac{\eta-2}{2(\eta+2)}$ and consider $\rho>\frac{2 \bar{R}}{1-2 q}$, then using (4) of Proposition 2.1, the 
asymptotic decay of $w$, and assumption $\left(H_{K}\right)$, we infer

$$
\begin{aligned}
\Phi\left(w\left(\cdot-\rho e_{1}\right)\right)(x)= & \frac{1}{4 \pi} \int_{\mathbb{R}^{3}} \frac{1}{|x-y|} K(y) w^{2}\left(y-\rho e_{1}\right) d y \\
= & \frac{1}{4 \pi}\left(\int_{\left\{y_{1}<\left(\frac{1}{2}-q\right) \rho\right\}} \frac{1}{|x-y|} K(y) w^{2}\left(y-\rho e_{1}\right) d y\right. \\
& \left.\quad+\int_{\left\{y_{1}>\left(\frac{1}{2}-q\right) \rho\right\}} \frac{1}{|x-y|} K(y) w^{2}\left(y-\rho e_{1}\right) d y\right) \\
\leq & c\left(e^{-2\left(\frac{1}{2}+q\right) \rho} \int_{\left\{y_{1}<\left(\frac{1}{2}-q\right) \rho\right\}} \frac{K(y)}{|x-y|} d y\right. \\
\leq & c e^{-(1+2 q) \rho}\left(\int_{\left\{y_{1}<\left(\frac{1}{2}-q\right) \rho\right\}} \frac{K(y)}{|x-y|} d y\right. \\
& \left.\quad+\int_{\left\{y_{1}>\left(\frac{1}{2}-q\right) \rho\right\}} \frac{w^{2}\left(y-\rho e_{1}\right)}{|x-y|} d y\right) \\
\leq & \left.c e^{-(1+2 q) \rho} . \quad \int_{1} \frac{1}{\mid x-q) \rho\}} d y\right)
\end{aligned}
$$

Then, a similar computation gives

$$
\begin{gathered}
\int_{\mathbb{R}^{3}} K(x) \phi_{w\left(-\rho e_{1}\right)}(x) w^{2}\left(x-\rho e_{1}\right) d x \leq c e^{-(1+2 q) \rho} \int_{\mathbb{R}^{3}} K(x) w^{2}\left(x-\rho e_{1}\right) d x \\
=c e^{-(1+2 q) \rho}\left(\int_{\left\{y_{1}<\left(\frac{1}{2}-q\right) \rho\right\}} K(x) w^{2}\left(x-\rho e_{1}\right) d y\right. \\
\left.+\int_{\left\{y_{1}>\left(\frac{1}{2}-q\right) \rho\right\}} K(x) w^{2}\left(x-\rho e_{1}\right) d y\right) \\
\leq c e^{-(1+2 q) \rho}\left(e^{-(1+2 q) \rho}\right)=c e^{-(2+4 q) \rho} .
\end{gathered}
$$

So the claim is obtained choosing $q_{\eta}=4 q$.

Lemma 3.7 Let $t_{\rho, z, s}$ and $\tau_{\rho, z, s}$ be the numbers defined in (3.13). There exists a constant $C>0$ such that

$$
t_{\rho, z, s}<C, \quad \forall \rho>0, \forall(z, s) \in \Sigma \times[0,1] .
$$


Furthermore

$$
t_{\rho, z, s}=\tau_{\rho, z, s}+o\left(\left[\rho e^{2 \rho}\right]^{-1}\right) .
$$

Proof First, let us observe that

$$
\left\|\bar{\psi}_{\rho}[z, s]\right\| \leq\left\|w\left(x-\rho e_{1}\right)\right\|+\|w(x-\rho z)\|=2\|w\|
$$

and

$$
\left|\bar{\psi}_{\rho}[z, s]\right|_{p+1} \geq \frac{1}{2}\left(\int_{B(0,1)} w^{p+1} d x\right)^{\frac{1}{p+1}}>0 .
$$

Therefore (3.15) comes straightly from (2.10).

Now, again using (2.10), we write

$$
\begin{aligned}
t_{\rho, z, s}^{p-1}= & \frac{\left\|\bar{\psi}_{\rho}[z, s]\right\|^{2}}{\left|\bar{\psi}_{\rho}[z, s]\right|_{p+1}^{p+1}-\int_{\mathbb{R}^{3}} \alpha\left|\bar{\psi}_{\rho}[z, s]\right|^{p+1} d x} \\
& +\frac{t_{\rho, z, s}^{2} \int_{\mathbb{R}^{3}} K(x) \phi_{\bar{\psi}_{\rho}[z, s]} \bar{\psi}_{\rho}^{2}[z, s] d x+\int_{\mathbb{R}^{3}} W(x) \bar{\psi}_{\rho}^{2}[z, s] d x}{\left|\bar{\psi}_{\rho}[z, s]\right|_{p+1}^{p+1}-\int_{\mathbb{R}^{3}} \alpha\left|\bar{\psi}_{\rho}[z, s]\right|^{p+1} d x} .
\end{aligned}
$$

By (3.15) and Lemma 3.6, we obtain at once

$$
t_{\rho, z, s} \int_{\mathbb{R}^{3}} K(x) \phi_{\bar{\psi}_{\rho}[z, s]}\left(\bar{\psi}_{\rho}[z, s]\right)^{2} d x=o\left(\left[\rho e^{2 \rho}\right]^{-1}\right),
$$

while, by Lemma 2.9 and assumptions $\left(H_{V}\right)$, and $\left(H_{a}\right)$ we get respectively

$$
\begin{gathered}
\int_{\mathbb{R}^{3}} W(x)\left(\bar{\psi}_{\rho}[z, s]\right)^{2} d x=o\left(\left[\rho e^{2 \rho}\right]^{-1}\right), \\
\int_{\mathbb{R}^{3}} \alpha(x)\left(\bar{\psi}_{\rho}[z, s]\right)^{p+1} d x=o\left(\left[\rho e^{2 \rho}\right]^{-1}\right) .
\end{gathered}
$$

Moreover, considering (3.18) and (3.17), we infer

$$
0<c \leq\left|\bar{\psi}_{\rho}[z, s]\right|_{p+1} \leq\left|w\left(x-\rho e_{1}\right)\right|_{p+1}+|w(x-\rho z)|_{p+1}=2|w|_{p+1},
$$

and

$$
0<\frac{1}{2}\left(\int_{B(0,1)}\left(|\nabla w|^{2}+w^{2}\right) d x\right)^{\frac{1}{2}} \leq\left\|\bar{\psi}_{\rho}[z, s]\right\| \leq 2\|w\| .
$$

Lastly, we obtain

$$
\frac{\left\|\bar{\psi}_{\rho}[z, s]\right\|^{2}}{\left|\bar{\psi}_{\rho}[z, s]\right|_{p+1}^{p+1}-\int_{\mathbb{R}^{3}} \alpha\left|\bar{\psi}_{\rho}[z, s]\right|^{p+1} d x}=\frac{\left\|\bar{\psi}_{\rho}[z, s]\right\|^{2}}{\left|\bar{\psi}_{\rho}[z, s]\right|_{p+1}^{p+1}}+o\left(\left[\rho e^{2 \rho}\right]^{-1}\right) .
$$


Then, inserting (3.20), (3.21), (3.23) in (3.19) we deduce

$$
t_{\rho, z, s}^{p-1}=\frac{\left\|\bar{\psi}_{\rho}[z, s]\right\|^{2}}{\left|\bar{\psi}_{\rho}[z, s]\right|_{p+1}^{p+1}}+o\left(\left[\rho e^{2 \rho}\right]^{-1}\right)=\tau_{\rho, z, s}^{p-1}+o\left(\left[\rho e^{2 \rho}\right]^{-1}\right)
$$

that gives (3.16), as desired.

q.e.d.

Proposition 3.8 There exists a number $\rho_{a}>0$ such that for all $\rho>\rho_{a}$

$$
\max \left\{I\left(\psi_{\rho}[z, s]\right):(z, s) \in \Sigma \times[0,1]\right\}<2 m_{\infty} .
$$

Proof In what follows we set $\varepsilon_{\rho}=\left[\rho e^{2 \rho}\right]^{-1}$.

By using (2.9) and (3) of Proposition 2.1, we write

$$
\begin{aligned}
I\left(\psi_{\rho}[z, s]\right)= & \left(\frac{1}{2}-\frac{1}{p+1}\right)\left\|t_{\rho, z, s} \bar{\psi}_{\rho}[z, s]\right\|^{2}+\left(\frac{1}{2}-\frac{1}{p+1}\right) t_{\rho, z, s}^{2} \int_{\mathbb{R}^{3}} W(x) \bar{\psi}_{\rho}^{2}[z, s] d x \\
& +\left(\frac{1}{4}-\frac{1}{p+1}\right) t_{\rho, z, s}^{4} \int_{\mathbb{R}^{3}} K(x) \phi_{\bar{\psi}_{\rho}[z, s]} \bar{\psi}_{\rho}^{2}[z, s] d x,
\end{aligned}
$$

from which using Lemmas 3.6 and 3.7, and equalities (3.21) and (2.14), we deduce that the following estimate holds true uniformly with respect to $(z, s) \in \Sigma \times[0,1]$ :

$$
\begin{aligned}
I\left(\psi_{\rho}[z, s]\right)= & \left(\frac{1}{2}-\frac{1}{p+1}\right)\left\|t_{\rho, z, s} \bar{\psi}_{\rho}[z, s]\right\|^{2}+o\left(\varepsilon_{\rho}\right) \\
= & \left(\frac{1}{2}-\frac{1}{p+1}\right)\left\|\tau_{\rho, z, s} \bar{\psi}_{\rho}[z, s]\right\|^{2}+\left(\frac{1}{2}-\frac{1}{p+1}\right)\left(t_{\rho, z, s}^{2}-\tau_{\rho, z, s}^{2}\right)\left\|\bar{\psi}_{\rho}[z, s]\right\|^{2} \\
& +o\left(\varepsilon_{\rho}\right) \\
= & \left(\frac{1}{2}-\frac{1}{p+1}\right)\left\|\tau_{\rho, z, s} \bar{\psi}_{\rho}[z, s]\right\|^{2}+o\left(\varepsilon_{\rho}\right)=I_{\infty}\left(\psi_{\infty, \rho}[z, s]\right)+o\left(\varepsilon_{\rho}\right) .
\end{aligned}
$$

Therefore, the claim is proved once one shows that some $\bar{c}>0$ exists such that

$$
I_{\infty}\left(\psi_{\infty, \rho}[z, s]\right) \leq 2 m_{\infty}-\bar{c} \varepsilon_{\rho}+o\left(\varepsilon_{\rho}\right) \quad \text { uniformly in }(z, s) \in \Sigma \times[0,1] .
$$

Now, let us estimate $I_{\infty}\left(\psi_{\infty, \rho}[z, s]\right)$ :

$$
\begin{aligned}
I_{\infty}\left(\psi_{\infty, \rho}[z, s]\right) & =I_{\infty}\left(\tau_{\rho, z, s} \bar{\psi}_{\rho}[z, s]\right)=\left(\frac{1}{2}-\frac{1}{p+1}\right)\left\|\tau_{\rho, z, s} \bar{\psi}_{\rho}[z, s]\right\|^{2} \\
& =\left(\frac{1}{2}-\frac{1}{p+1}\right)\left(\frac{\bar{\psi}_{\rho}[z, s] \|^{2}}{\left|\bar{\psi}_{\rho}[z, s]\right|_{p+1}^{2}}\right)^{\frac{p+1}{p-1}} .
\end{aligned}
$$


By direct computation, taking into account that $w$ solves $\left(P_{\infty}\right)$, we obtain

$$
\left\|\bar{\psi}_{\rho}[z, s]\right\|^{2}=\left[(1-s)^{2}+s^{2}\right]\|w\|^{2}+2 s(1-s) \int_{\mathbb{R}^{3}} w^{p}\left(x-\rho e_{1}\right) w(x-\rho z) d x,
$$

and, by Lemma 2.9, we can assert that $c_{1}>0$ exists such that

$$
\lim _{\rho \rightarrow+\infty} \frac{1}{\varepsilon_{\rho}} \int_{\mathbb{R}^{3}} w^{p}\left(x-\rho e_{1}\right) w(x-\rho z) d x=\lim _{\rho \rightarrow+\infty} \frac{1}{\varepsilon_{\rho}} \int_{\mathbb{R}^{3}} w\left(x-\rho e_{1}\right) w^{p}(x-\rho z) d x=c_{1} .
$$

Thus, inserting (3.27) in (3.26) we deduce

$$
\left\|\bar{\psi}_{\rho}[z, s]\right\|^{2}=\left[(1-s)^{2}+s^{2}\right]\|w\|^{2}+2 s(1-s) c_{1} \varepsilon_{\rho}+o\left(\varepsilon_{\rho}\right) .
$$

On the other hand, applying Lemma 2.8 and using (3.27) we get

$$
\begin{aligned}
\left|\bar{\psi}_{\rho}[z, s]\right|_{p+1}^{p+1} & =\int_{\mathbb{R}^{3}}\left[(1-s) w\left(x-\rho e_{1}\right)+s w(x-\rho z)\right]^{p+1} d x \\
& \geq\left[(1-s)^{p+1}+s^{p+1}\right]|w|_{p+1}^{p+1}+p\left[(1-s)^{p} s+(1-s) s^{p}\right] c_{1} \varepsilon_{\rho}+o\left(\varepsilon_{\rho}\right) .
\end{aligned}
$$

Combining estimates (3.28) and (3.29) and taking advantage of a Taylor expansion, we then obtain for all $s \in[0,1]$ and $z \in \Sigma$

$$
\begin{aligned}
\frac{\left\|\bar{\psi}_{\rho}[z, s]\right\|^{2}}{\left|\bar{\psi}_{\rho}[z, s]\right|_{p+1}^{2}} & \leq \frac{\left[(1-s)^{2}+s^{2}\right]\|w\|^{2}+2 s(1-s) c_{1} \varepsilon_{\rho}+o\left(\varepsilon_{\rho}\right)}{\left(\left[(1-s)^{p+1}+s^{p+1}\right]|w|_{p+1}^{p+1}+p\left[(1-s)^{p} s+(1-s) s^{p}\right] c_{1} \varepsilon_{\rho}+o\left(\varepsilon_{\rho}\right)\right)^{\frac{2}{p+1}}} \\
& =\frac{(1-s)^{2}+s^{2}}{\left((1-s)^{p+1}+s^{p+1}\right)^{\frac{2}{p+1}}} \frac{\|w\|^{2}}{|w|_{p+1}^{2}}+\gamma(s) \varepsilon_{\rho}+o\left(\varepsilon_{\rho}\right)
\end{aligned}
$$

where

$$
\gamma(s)=\frac{2 s(1-s) c_{1}}{\left[(1-s)^{p+1}+s^{p+1}\right]^{\frac{2}{p+1}}} \frac{1}{|w|_{p+1}^{2}}\left[1-\frac{p}{p+1} \frac{(1-s)^{2}+s^{2}}{(1-s)^{p+1}+s^{p+1}}\left[(1-s)^{p-1}+s^{p-1}\right]\right] .
$$

Now, let us observe that $\gamma(1 / 2)<0$, because $p>1$, so taking into account (2.15), we can assert the existence of a neighbourhood $\mathcal{I}(1 / 2)$ of $1 / 2$ and of a constant $\bar{c}>0$ such that

$$
\begin{aligned}
I_{\infty}\left(\psi_{\infty, \rho}[z, s]\right) & \leq\left(\frac{1}{2}-\frac{1}{p+1}\right)\left[2^{\frac{p-1}{p+1}} \frac{\|w\|^{2}}{|w|_{p+1}^{2}}\right]^{\frac{p+1}{p-1}}-\bar{c} \varepsilon_{\rho}+o\left(\varepsilon_{\rho}\right) \\
& =2\left(\frac{1}{2}-\frac{1}{p+1}\right)|w|_{p+1}^{p+1}-\bar{c} \varepsilon_{\rho}+o\left(\varepsilon_{\rho}\right) \\
& =2 m_{\infty}-\bar{c} \varepsilon_{\rho}+o\left(\varepsilon_{\rho}\right) \quad \forall z \in \Sigma, \quad \forall s \in \mathcal{I}(1 / 2) .
\end{aligned}
$$


Similar computations show that

$$
\begin{gathered}
\lim _{\rho \rightarrow+\infty} \max \left\{I_{\infty}\left(\psi_{\infty, \rho}[z, s]\right): z \in \Sigma, s \in[0,1] \backslash \mathcal{I}(1 / 2)\right\} \\
=\max \left\{\left(\frac{(1-s)^{2}+s^{2}}{\left[(1-s)^{p+1}+s^{p+1}\right]^{\frac{2}{p+1}}}\right)^{\frac{p+1}{p-1}} m_{\infty}: s \in[0,1] \backslash \mathcal{I}\left(\frac{1}{2}\right)\right\}<2 m_{\infty} .
\end{gathered}
$$

(3.31) and (3.32) give (3.24) completing the proof.

q.e.d.

Proof of Theorem 1.1 Let us fix $\rho>\max \left\{\rho_{b}, \rho_{a}\right\}$ being $\rho_{b}$ and $\rho_{a}$ the numbers whose existence is claimed in Propositions 3.5 and 3.8, respectively. Let us set

$$
\mathcal{A}=\max _{\Sigma \times[0,1]} I\left(\psi_{\rho}[z, s]\right), \quad \mathcal{T}=\max _{\Sigma} I\left(\psi_{\rho}[z, 1]\right) .
$$

Moreover in what follows let us denote by $I^{c}:=\{u \in \mathcal{N}: I(u) \leq c\}$.

Applying Propositions 3.1, 3.5, 3.4, and 3.8, the following chain of inequalities is obtained

$$
m_{\infty}<\mathcal{T}<\mathcal{B}_{0} \leq \mathcal{A}<2 m_{\infty}
$$

We claim that there exists a critical value in $\left[\mathcal{B}_{0}, \mathcal{A}\right]$. Assume, by contradiction, that this is not the case. Then, since by Corollary 3.2 the Palais-Smale condition holds in $\left(m_{\infty}, 2 m_{\infty}\right)$, we can apply usual deformation arguments (see f.i.[21]) and assert the existence of a number $\delta>0$ and a continuous function

$$
\sigma: I^{\mathcal{A}} \rightarrow I^{\mathcal{B}_{0}-\delta}
$$

such that $\mathcal{B}_{0}-\delta>\mathcal{T}$ and

$$
\sigma(u)=u \quad \forall u \in I^{\mathcal{B}_{0}-\delta} .
$$

Thus

$$
0 \notin\left(\beta \circ \sigma \circ \psi_{\rho}\right)(\Sigma \times[0,1]) .
$$

On the other hand, considering the choice of $\rho$, Proposition 3.4, the inclusion $\psi_{\rho}(\Sigma \times$ $\{1\}) \subseteq I^{\mathcal{T}}$, and the continuity of $\beta, \sigma, \psi_{\rho}$ we deduce, thanks to the invariance of topological degree by homotopy, $0 \neq d\left(\mathcal{G}_{\rho}, \Sigma \times[0,1), 0\right)=d\left(\beta \circ \sigma \circ \psi_{\rho}, \Sigma \times[0,1), 0\right)$, being, as in Proposition 3.4, $\mathcal{G}_{\rho}(z, s)=(1-s) \rho e_{1}+s \rho z$.

Therefore, we conclude that

$$
\exists(\tilde{z}, \tilde{s}) \in \Sigma \times[0,1): \quad\left(\beta \circ \sigma \circ \psi_{\rho}\right)(\tilde{z}, \tilde{s})=0
$$

reaching a contradiction and proving the claim. 
Now, to complete the proof we only must show that the solution of (2.3) corresponding to the critical level existing in the interval $\left(m_{\infty}, 2 m_{\infty}\right)$ is a constant sign solution. To this end we show that if $u$ is a solution of $(2.3) u^{+} \neq 0$ and $u^{-} \neq 0$, then $I(u) \geq 2 m_{\infty}$. The argument is analogous to that of Remark 2.6. Since one easily sees that $u^{+} \in \mathcal{N}$ and $u^{-} \in \mathcal{N}$ then, by Proposition 3.1, $I\left(u^{+}\right) \geq m_{\infty}$ and $I\left(u^{-}\right) \geq m_{\infty}$, from which $I(u)=I\left(u^{+}\right)+I\left(u^{-}\right) \geq 2 m_{\infty}$ follows.

Acknowledgement. The authors have been supported by the "Gruppo Nazionale per l'Analisi Matematica, la Probabilità e le loro Applicazioni (GNAMPA)" of the Istituto Nazionale di Alta Matematica (INdAM).

\section{References}

[1] A.Ambrosetti, On Schrödinger-Poisson systems, Milan J. Math. 76 (2008), 257-274.

[2] A. Ambrosetti - D. Ruiz, Multiple bound states for Schrödinger- Poisson problems, Comm. Contemp. Math. 10 (2008), 391-404.

[3] A. Azzollini - A. Pomponio, Ground state solutions for the nonlinear SchrödingerMaxwell equations, J.Math. Anal. Appl. 345 (2008), 90-108.

[4] A. Bahri - Y.Y. Li, On a min-max procedure for the existence of a positive solution for certain scalar field equations in $R^{N}$, Rev. Mat. Iberoamericana 6 (1990), 1-15.

[5] A. Bahri - P.L.Lions, On the existence of a positive solution of semilinear elliptic equations in unbounded domains Ann. Inst. H. Poincaré Anal. Non Linéaire 14 (1997), 365413.

[6] V. Benci - D. Fortunato, An eigenvalue problem for the Schrödinger-Maxwell equations Topol. Methods Nonlinear Anal. 99 (1998), 283-293.

[7] V. Benci - D. Fortunato, Solitary waves of the nonlinear Klein-Gordon equation coupled with Maxwell equations, Rev. Math. Phys. 14 (2002), 409-420.

[8] V. Benci - D. Fortunato, Solitons in Schrödinger-Maxwell equations J. Fixed Point Theory Appl. 15 (2014)101-132.

[9] H. Berestycki - P.L. Lions, Nonlinear scalar field equations II. Existence of infinitely many solutions, Arch. Rational Mech. Anal. 82 (1983), no. 4, 347-375.

[10] D. Bonheure - C. Mercuri, Embedding theorems and existence results for nonlinear Schrd̈̈nger-Poisson systems with unbounded and vanishing potentials, J. Differential Equations 251 (2011), no. 4-5, 1056-1085. 
[11] D. Bonheure - J. Di Cosmo - C. Mercuri, Concentration on circles for nonlinear Schrödinger-Poisson systems with unbounded potentials vanishing at infinity, Commun. Contemp. Math. 14 (2012), no. 2, 1250009, 31 pp..

[12] G. Cerami, Some nonlinear elliptic problems in unbounded domains, Milan J. Math. 74 (2006), 47-77.

[13] G. Cerami - D. Passaseo, Existence and multiplicity results for semilinear elliptic Dirichlet problems in exterior domains, Nonlinear Anal. 24 (1995), no. 11, 1533-1547.

[14] G. Cerami - D. Passaseo, The effect of concentrating potentials in some singularly perturbed problems, Calc. Var. PDE 17 (2003), 257-281.

[15] G. Cerami - G. Vaira, Positive solutions for some non-autonomous Schrödinger-Poisson systems, J. Differential Equations 248 (2010), no. 3, 521-543.

[16] M.Furtado - L.A Maia - E.S.Medeiros, A note on the existence of a positive solution for a non-autonomous Schrödinger-Poisson system, Analysis and Topology in Nonlinear Differential Equations, Progress in Nonlinear Differential Equations and Their Applications, 85 (2014) Springer Int. Publ., 277-286.

[17] B. Gidas - W.M. Ni - L. Nirenberg, Symmetry and related properties via the maximum principle, Comm. Math. Phys. 68 (1979), no. 3, 209-243.

[18] M.K. Kwong, Uniqueness of positive solutions of $\Delta u-u+u^{p}=0$ in $\mathbb{R}^{N}$, Arch. Rat. Mech. Anal. 105 (1989), 243-266.

[19] C. Mercuri - V. Moroz - J. Van Schaftingen, Groundstates and radial solutions to nonlinear Schrödinger-Poisson-Slater equations at the critical frequency, preprint arXiv: 1507.02837 .

[20] D. Ruiz, The Schrödinger-Poisson equation under the effect of a nonlinear local term, J. functional anal. 237 (2006), 655-674.

[21] M. Struwe, Variational Methods - Applications to nonlinear PDE and Hamiltonian Systems, 4th edition. Ergeb. Math. Grenzgeb. 2008 Springer-Verlag, Berlin.

[22] T. Weth, Energy bounds for entire nodal solutions of autonomous superlinear equations, Calc. Var. Partial Differential Equations 27 (2006), no. 4, 421-437. 